\title{
Three-dimensional telegrapher's equation and its fractional generalization
}

\author{
Jaume Masoliver ${ }^{*}$ \\ Department of Condensed Matter Physics and Institute of Complex Systems (UBICS), University of Barcelona, Catalonia, Spain
}

(Received 15 May 2017; published 1 August 2017)

\begin{abstract}
We derive the three-dimensional telegrapher's equation out of a random walk model. The model is a threedimensional version of the multistate random walk where the number of different states form a continuum representing the spatial directions that the walker can take. We set the general equations and solve them for isotropic and uniform walks which finally allows us to obtain the telegrapher's equation in three dimensions. We generalize the isotropic model and the telegrapher's equation to include fractional anomalous transport in three dimensions.
\end{abstract}

DOI: 10.1103/PhysRevE.96.022101

\section{INTRODUCTION}

Within the vast and complex field of transport of particles through continuous media, diffusion is surely the most used approach for modeling the whole process. The main reason lies in the fact that diffusion provides one of the simplest ways of reproducing many qualitative features of transport as well as giving reasonable quantitative estimates [1].

When the moving particle is not driven by any external field, the diffusive approximation reduces the transport equation (usually a rather complicated and nonlinear integrodifferential equation with an imperfectly known scattering kernel [2]) to the much simpler diffusion equation,

$$
\frac{\partial p}{\partial t}=D \nabla^{2} p
$$

where $p(\mathbf{r}, t)$ is the probability density function of the diffusing particle to be at $\mathbf{r}$ at time $t$ and $D$ is the diffusion coefficient. A major characteristic of any diffusion process is that the mean square deviation grows linearly with time,

$$
\left\langle|\Delta \mathbf{r}(t)|^{2}\right\rangle=D t,
$$

where $\Delta \mathbf{r}(t)=\mathbf{r}(t)-\langle\mathbf{r}(t)\rangle$. A second major characteristic is the Gaussian character of the free diffusion process. Thus the solution to Eq. (1) in unbounded space and assuming the particle is initially at the origin $p(\mathbf{r}, 0)=\delta(\mathbf{r})$ reads

$$
p(\mathbf{r}, t)=\frac{1}{(4 \pi D t)^{3 / 2}} e^{-r^{2} / 4 D t} .
$$

Let us observe that the form of this solution shows that for any $t>0$ the function $p(\mathbf{r}, t)>0$ is strictly positive for any value of $r=|\mathbf{r}|$. As a result, there is a nonzero probability of finding the particle arbitrarily far away from the initial position. In other words, the diffusion model allows for the possibility of particles traveling at arbitrary velocities, even larger than the speed of light in vacuum. The diffusive approximation is, therefore, not compatible with relativity [3].

There are further limitations of the diffusion approximation. Thus, in situations, such as those of photon migration through continuous media, one finds the inability of the diffusion equation to account for ballistic motion as well as inaccuracies near interfaces and in thin samples. Also in the description of

\footnotetext{
*jaume.masoliver@ub.edu
}

early-time effects when the flux of ballistic particles may not be negligible which may result in anisotropic scattering along the forward direction [1,4-6].

Although there are no unambiguous and widely accepted relativistic generalizations of the diffusion equation, it has been suggested by a number of researchers from different fields that one of the simplest generalizations (still retaining significant diffusive properties but characterized by a finite propagation speed as well as considering possible fluxes of ballistic particles) is the telegrapher's equation (TE) [4-18]. The equation first appeared in the nineteen century with the works of Kelvin and Heaviside related to the analysis of the distortion and dissipation of electromagnetic waves in telegraph lines [9]. In this electromagnetic context the three-dimensional telegrapher's equation is derived directly from combining Maxwell's equations for homogeneous media $[1,9]$.

The generic form of the TE is

$$
\frac{\partial^{2} p}{\partial t^{2}}+\frac{1}{\tau} \frac{\partial p}{\partial t}=v^{2} \nabla^{2} p,
$$

where $\tau>0$ is a characteristic time and $v>0$ is a characteristic speed. It is a hyperbolic equation which as $\tau \rightarrow \infty$ with $v$ fixed becomes the wave equation whereas as $\tau \rightarrow 0$ and $v \rightarrow \infty$ with $v^{2} \tau \rightarrow D$ finite it turns into the diffusion equation. Equation (4) thus possesses wave and diffusion features, and it describes "diffusion with finite propagation velocity" $[9,11]$.

In the context of transport theory, the three-dimensional TE is the so-called $P_{1}$ approximation to the full transport equation for which the basic assumption is that the change in direction due to a single scattering event is small $[1,2,10,19]$. Other approaches suppose phenomenological generalizations where a three-dimensional TE is postulated by assuming the same form as the one-dimensional TE but with numerical corrections in the coefficients which guarantee correct ballistic and diffusive behaviors in three dimensions [4-6]. In one recent approach [20] such a generalization consists of a convenient modification of the continuity equation for the probability current. The model is, however, limited to a discrete number of directions which restricts possible applications to transport processes.

Within this background, a number of works have traditionally attempted to obtain the TE from random walk models since they try to reproduce the microscopic (or, at 
least, mesoscopic) mechanism of the transport process. These models are modifications of the ordinary random walk because the latter, in the so-called "fluid limit" (see below), leads to the diffusion equation but not to the telegrapher's equation [1,9].

Despite that in one dimension the TE is readily achieved from the persistent random walk on the line $[1,18,21]$, in higher dimensions obtaining the TE from microscopic models encounters serious difficulties. The main reason lying in the difficulty of generalizing persistence in dimensions higher than one [22-28].

One chief objective of the present paper is to arrive at the three-dimensional TE out of a random walk model. To this end we propose and develop a continuous version of a threedimensional multistate random walk with a continuum in the number of states. The model may be seen as a generalization of the persistent random walk in three dimensions, and it has been inspired by previous work on continuous-valued noise [29]. As we will see the model can be solved for homogeneous and isotropic walks which, in turn, finally leads to the threedimensional TE.

For more than two decades, so-called "anomalous transport" and "anomalous diffusion" have been the object of intense research with countless applications in many areas of physics, chemistry, and natural and socioeconomic sciences. There is immense literature on the subject, and for complete reports we may cite Refs. [30-39] among many others. The concept first emerged from the theory of random processes, specifically from continuous time random walks [40-42], and it was first applied to diffusion of charge carriers in organic semiconductors by Scher and Montroll in Refs. [43,44].

Anomalous transport arises in extremely disordered systems, such as random media and fractal structures [45], and its most distinctive characteristic is that the mean square deviation follows the asymptotic law [31,32]:

$$
\left\langle|\Delta \mathbf{r}(t)|^{2}\right\rangle \sim t^{\alpha}
$$

$(t \rightarrow \infty)$, where $\alpha>0$ is any positive real number. The range of $0<\alpha<1$ describes subdiffusion, $\alpha=1$ corresponds to (normal) diffusive transport, and $\alpha>1$ describes superdiffusion. Within the diffusive approximation and in the force-free case, the anomalous transport process is described by a fractional diffusion equation, which can be written as

$$
\frac{\partial^{\alpha} p}{\partial t^{\alpha}}=D \nabla^{2 \gamma} p
$$

$(0<\alpha \leqslant 1,0<\gamma \leqslant 1)$, where $\partial^{\alpha} / \partial t^{\alpha}$ is the fractional Caputo derivative and $\nabla^{2 \gamma}$ is the Riesz-Feller fractional Laplacian (see Sec. VI). The mathematical properties of the solutions to the fractional diffusion equation have been studied thoroughly and very clearly exposed by Mainardi and collaborators [46-48], and we refer the reader to the works of these authors for more information.

As mentioned above, the formulation of anomalous transport originally was devised out of the continuous time random walk formalism $[43,44]$. As a result, the derivations of Eq. (6) are based mostly on this formalism. Even though there also exist alternative approaches based on master equations or (fractional) Chapman-Kolmogorov expansions [32].

Similar to the ordinary diffusion equation (1), the fractional equation (6) ignores possible changes in the dynamics of the diffusing particle as time increases. We recall that these changes account for ballistic motion and anisotropic scattering (among others) that are relevant in a number of experimental settings [49]. As we have seen, the TE explains some of these characteristics of transport which imply, in particular, the transition from ballistic to diffusive motion with the increase in time.

In a recent work [50] we have presented a derivation of the fractional telegrapher's equation (FTE) in one dimension which is based on a fractional generalization of the persistent random walk on the line. We also have shown there that a simple and direct three-dimensional generalization of the one-dimensional persistent random walk leads to inconsistent results. A second chief objective of this paper is, therefore, obtaining a three-dimensional FTE out of a fractional generalization of our continuous and isotropic random walk model.

The paper is organized as follows. In Sec. II we present the continuous multistate random walk in three dimensions, which is a generalization of the multistate random walk allowing for a continuous number of states. In Sec. III we study the special case of an isotropic and homogeneous walk. In Sec. IV we derive, out of the isotropic walk, the three-dimensional TE. In Sec. V we generalize the isotropic walk to include a fractional version of it. From this generalization, we derive the three-dimensional fractional telegrapher's equation in Sec. VI. Some concluding remarks are in Sec. VII.

\section{CONTINUOUS MULTISTATE RANDOM WALK IN THREE DIMENSIONS}

In this section we present a microscopic model for the transport of particles in continuous media. The model is based on a generalization of multistate random walks (see Ref. [51] and references therein) and assumes a continuum in the number of states [29]. In the standard formulation of multistate random walks, the walker can be in a discrete number of internal states, and the transition between different states is random and governed by a given transition matrix that generally is supposed to be Markovian. The usual formulation also assumes that the walker moves on a line. In order to model transport of particles we will generalize the multistate random walk in two key features: (i) We will assume that the walker (i.e., the particle) moves in three dimensions, and (ii) it can have internal states defined on a continuous set of values.

Suppose a random walker moving in the three-dimensional space. At any instant in time the walker moves along a straight line with a direction determined by the solid angle $\boldsymbol{\Omega}=(\theta, \varphi)$, where $\theta$ is the polar angle and $\varphi$ is the azimuthal angle. The particular direction in which the particle is moving constitutes the "internal state," and, since all possible directions form a continuous and denumerable set, we will deal with a continuous multistate random walk.

The walker shifts direction randomly. Hence the duration of the motion along a given direction $\boldsymbol{\Omega}$, which we call sojourn, is a random variable determined by a probability density function (PDF) denoted by $\psi(t \mid \boldsymbol{\Omega})$. The probability that the duration of a given sojourn is greater than $t$ will thus be given by

$$
\Psi(t \mid \boldsymbol{\Omega})=\int_{t}^{\infty} \psi\left(t^{\prime} \mid \boldsymbol{\Omega}\right) d t^{\prime} .
$$


Let us further indicate by $h(\mathbf{r}, t \mid \boldsymbol{\Omega})$ the joint PDF for the displacement in a single sojourn along direction $\boldsymbol{\Omega}$ to be equal to $\mathbf{r}$ and the sojourn duration to equal $t$. In addition, let $H(\mathbf{r}, t \mid \boldsymbol{\Omega})$ be the probability density for the displacement to be $\mathbf{r}$ in a single sojourn when the total sojourn time is greater than $t$. Notice that the duration $\operatorname{PDF} \psi(t \mid \boldsymbol{\Omega})$ is the time marginal density of $h(\mathbf{r}, t \mid \boldsymbol{\Omega})$,

$$
\int_{\mathbb{R}^{3}} h(\mathbf{r}, t \mid \boldsymbol{\Omega}) d^{3} \mathbf{r}=\psi(t \mid \boldsymbol{\Omega}),
$$

whereas

$$
\int_{\mathbb{R}^{3}} H(\mathbf{r}, t \mid \boldsymbol{\Omega}) d^{3} \mathbf{r}=\Psi(t \mid \boldsymbol{\Omega}) .
$$

At the end of a given sojourn the walker, which is supposed to move along $\boldsymbol{\Omega}^{\prime}$, switches to direction $\boldsymbol{\Omega}$. We denote by $\beta\left(\boldsymbol{\Omega} \mid \boldsymbol{\Omega}^{\prime}\right)$ the scattering kernel, that is, the PDF for the transition $\boldsymbol{\Omega}^{\prime} \rightarrow \boldsymbol{\Omega}$. In this way the probability that a single scattering changes the direction of the walker from $\boldsymbol{\Omega}^{\prime}$ to a direction falling somewhere inside the angular region $(\boldsymbol{\Omega}, \boldsymbol{\Omega}+d \boldsymbol{\Omega})$ is given by

$$
\operatorname{Prob}\left\{\boldsymbol{\Omega}^{\prime} \rightarrow(\boldsymbol{\Omega}, \boldsymbol{\Omega}+d \boldsymbol{\Omega})\right\}=\beta\left(\boldsymbol{\Omega} \mid \boldsymbol{\Omega}^{\prime}\right) d^{2} \boldsymbol{\Omega},
$$

where $d \boldsymbol{\Omega}=(d \theta, d \varphi)$ and

$$
d^{2} \boldsymbol{\Omega}=\sin \theta d \theta d \varphi
$$

denotes the surface element on the sphere of the unit radius.

Let us observe that in this model the walker after each sojourn has a nonvanishing probability of traveling in the previous direction. In those cases where this probability is higher than $1 / 2$, the walk is persistent in moving along a given direction. We may well say that the model constitutes a three-dimensional generalization of the persistent random walk [42].

Our objective is to find the PDF for the walker to be at $\mathbf{r}$ at time $t$. We denote this density by $p(\mathbf{r}, t)$. It can be written as

$$
p(\mathbf{r}, t)=\int p(\mathbf{r}, \boldsymbol{\Omega}, t) d^{2} \boldsymbol{\Omega},
$$

where $p(\mathbf{r}, \boldsymbol{\Omega}, t)$ is the joint PDF for the random walker to be at $\mathbf{r}$ at time $t$ and moving in direction $\boldsymbol{\Omega}$ and the integral extends over all possible directions.

A first step in the calculation of $p(\mathbf{r}, \boldsymbol{\Omega}, t)$ relies on the knowledge of an intermediate function denoted by $\rho(\mathbf{r}, \boldsymbol{\Omega}, t)$ and defined as

$$
\begin{aligned}
\rho(\mathbf{r}, \boldsymbol{\Omega}, t) d^{3} \mathbf{r} d t= & \operatorname{Prob}\{\text { a sojourn in direction } \boldsymbol{\Omega} \text { ends in } \\
& \text { the region }(\mathbf{r}, \mathbf{r}+d \mathbf{r}) \text { at }(t, t+d t)\} .
\end{aligned}
$$

Note that this density describes the state of the process at the "scattering points" where the direction of the walker changes. A standard renewal argument shows that the auxiliary density obeys the following integral equation:

$$
\begin{aligned}
\rho(\mathbf{r}, \boldsymbol{\Omega}, t)= & \beta(\boldsymbol{\Omega}) h(\mathbf{r}, t \mid \boldsymbol{\Omega})+\int \beta\left(\boldsymbol{\Omega} \mid \boldsymbol{\Omega}^{\prime}\right) d^{2} \boldsymbol{\Omega}^{\prime} \int_{0}^{t} d t^{\prime} \\
& \times \int_{\mathbb{R}^{3}} h\left(\mathbf{r}-\mathbf{r}^{\prime}, t-t^{\prime} \mid \boldsymbol{\Omega}\right) \rho\left(\mathbf{r}^{\prime}, \boldsymbol{\Omega}^{\prime}, t^{\prime}\right) d^{3} \mathbf{r}^{\prime},
\end{aligned}
$$

where $\beta(\boldsymbol{\Omega})$ is the probability that the process starts moving in direction $\boldsymbol{\Omega}$. The renewal reasoning behind Eq. (13) simply says that, if a scattering event occurs at time $t$, it must be either the first one (after the initial one at $t=0$ ) this described by the first term on the right hand side, or else an earlier scattering $\boldsymbol{\Omega}^{\prime} \rightarrow \boldsymbol{\Omega}$, governed by the density $\beta\left(\boldsymbol{\Omega} \mid \boldsymbol{\Omega}^{\prime}\right)$, occurred at time $t^{\prime}<t$ when the walker was at $\mathbf{r}^{\prime}$ and no further change in direction occurred during the time interval $t-t^{\prime}$. All of this integrated over all possible directions $\boldsymbol{\Omega}^{\prime}$, positions $\mathbf{r}^{\prime} \in \mathbb{R}^{3}$, and intermediate times $t^{\prime}$.

Once we know the auxiliary density $\rho(\mathbf{r}, \boldsymbol{\Omega}, t)$, the PDF $p(\mathbf{r}, \boldsymbol{\Omega}, t)$ for the walker to be at $\mathbf{r}$ at time $t$ while moving in direction $\boldsymbol{\Omega}$ is

$$
\begin{aligned}
p(\mathbf{r}, \boldsymbol{\Omega}, t)= & \beta(\boldsymbol{\Omega}) H(\mathbf{r}, t \mid \boldsymbol{\Omega})+\int \beta\left(\boldsymbol{\Omega} \mid \boldsymbol{\Omega}^{\prime}\right) d^{2} \boldsymbol{\Omega}^{\prime} \int_{0}^{t} d t^{\prime} \\
& \times \int_{\mathbb{R}^{3}} H\left(\mathbf{r}-\mathbf{r}^{\prime}, t-t^{\prime} \mid \boldsymbol{\Omega}\right) \rho\left(\mathbf{r}^{\prime}, \boldsymbol{\Omega}^{\prime}, t^{\prime}\right) d^{3} \mathbf{r}^{\prime} .
\end{aligned}
$$

The reasoning behind this equation is similar to that following Eq. (13). Thus the displacement of the walker is either within the first sojourn, this given by $\beta H$ on the right hand side, or else an earlier change in direction occurred at time $t^{\prime}<t$ while the walker was at position $\mathbf{r}^{\prime}$ and the time interval to the next scattering event exceeded $t-t^{\prime}$.

The degree of difficulty in solving the integral equation (13) is reduced by using the Fourier-Laplace transform,

$$
\hat{\tilde{\rho}}(\boldsymbol{\omega}, \boldsymbol{\Omega}, s)=\int_{0}^{\infty} e^{-s t} d t \int_{\mathbb{R}^{3}} e^{i \boldsymbol{\omega} \cdot \mathbf{r}} \rho(\mathbf{r}, \boldsymbol{\Omega}, t) d^{3} \mathbf{r} .
$$

Indeed, transforming Eqs. (13) and (14) yields

$$
\begin{aligned}
\hat{\tilde{\rho}}(\boldsymbol{\omega}, \boldsymbol{\Omega}, s)= & \hat{\tilde{h}}(\boldsymbol{\omega}, s \mid \boldsymbol{\Omega}) \\
& \times\left[\beta(\boldsymbol{\Omega})+\int \beta\left(\boldsymbol{\Omega} \mid \boldsymbol{\Omega}^{\prime}\right) \hat{\tilde{\rho}}\left(\boldsymbol{\omega}, \boldsymbol{\Omega}^{\prime}, s\right) d^{2} \boldsymbol{\Omega}^{\prime}\right],
\end{aligned}
$$

and

$$
\begin{aligned}
\hat{\tilde{p}}(\boldsymbol{\omega}, \boldsymbol{\Omega}, s)= & \hat{\tilde{H}}(\boldsymbol{\omega}, s \mid \boldsymbol{\Omega}) \\
& \times\left[\beta(\boldsymbol{\Omega})+\int \beta\left(\boldsymbol{\Omega} \mid \boldsymbol{\Omega}^{\prime}\right) \hat{\tilde{\rho}}\left(\boldsymbol{\omega}, \boldsymbol{\Omega}^{\prime}, s\right) d^{2} \boldsymbol{\Omega}^{\prime}\right] .
\end{aligned}
$$

From Eq. (15) we see that

$$
\int \beta\left(\boldsymbol{\Omega} \mid \boldsymbol{\Omega}^{\prime}\right) \hat{\tilde{\rho}}\left(\boldsymbol{\omega}, \boldsymbol{\Omega}^{\prime}, s\right) d^{2} \boldsymbol{\Omega}^{\prime}=\frac{\hat{\tilde{\rho}}(\boldsymbol{\omega}, \boldsymbol{\Omega}, s)}{\hat{\tilde{h}}(\boldsymbol{\omega}, s \mid \boldsymbol{\Omega})}-\beta(\boldsymbol{\Omega}),
$$

which after substituting into Eq. (16) yields a rather simple relation between $\hat{\tilde{p}}(\boldsymbol{\omega}, \boldsymbol{\Omega}, s)$ and $\hat{\tilde{\rho}}(\boldsymbol{\omega}, \boldsymbol{\Omega}, s)$,

$$
\hat{\tilde{p}}(\boldsymbol{\omega}, \boldsymbol{\Omega}, s)=\frac{\hat{\tilde{H}}(\boldsymbol{\omega}, \boldsymbol{\Omega}, s)}{\hat{\tilde{h}}(\boldsymbol{\omega}, s \mid \boldsymbol{\Omega})} \hat{\tilde{\rho}}(\boldsymbol{\omega}, \boldsymbol{\Omega}, s) .
$$

Finally, plugging Eq. (17) into the Fourier-Laplace transform of Eq. (12) yields

$$
\hat{\tilde{p}}(\boldsymbol{\omega}, s)=\int \frac{\hat{\tilde{H}}(\boldsymbol{\omega}, \boldsymbol{\Omega}, s)}{\hat{\tilde{h}}(\boldsymbol{\omega}, s \mid \boldsymbol{\Omega})} \hat{\tilde{\rho}}(\boldsymbol{\omega}, \boldsymbol{\Omega}, s) d^{2} \boldsymbol{\Omega} .
$$

The solution of the integral equation (15) for $\hat{\tilde{\rho}}(\boldsymbol{\omega}, \boldsymbol{\Omega}, s)$ and its subsequent use in Eq. (18) summarize the main results of the model and furnish a convenient starting point for numerical work in the most general case when no further simplifications can be performed. 


\section{Independent scattering}

In order to proceed further we should specify some form for the scattering kernel $\beta\left(\boldsymbol{\Omega} \mid \boldsymbol{\Omega}^{\prime}\right)$. For this purpose we will consider the case in which the direction is randomized at the end of each sojourn independent of its previous value. In this way the PDF for the transition $\boldsymbol{\Omega}^{\prime} \rightarrow \boldsymbol{\Omega}$,

$$
\beta\left(\boldsymbol{\Omega} \mid \boldsymbol{\Omega}^{\prime}\right)=\beta(\boldsymbol{\Omega})
$$

is independent of $\boldsymbol{\Omega}^{\prime}$. The scattering process is thus an independent random process in the change in direction. Let us incidentally mention that this model is similar in spirit to that of Gordon in his study of rotational relaxation in fluids wherein the angular momentum is randomized at each collision [52]. In the context of fluctuations in laser fields the model corresponds to the so-called Burshtein model $[53,54]$.

When the scattering kernel has the form given by Eq. (19), Eqs. (15) and (16) reduce to

$$
\hat{\tilde{\rho}}(\boldsymbol{\omega}, \boldsymbol{\Omega}, s)=\beta(\boldsymbol{\Omega}) \hat{\tilde{h}}(\boldsymbol{\omega}, s \mid \boldsymbol{\Omega})\left[1+\int \hat{\tilde{\rho}}\left(\boldsymbol{\omega}, \boldsymbol{\Omega}^{\prime}, s\right) d^{2} \boldsymbol{\Omega}^{\prime}\right],
$$

and

$$
\hat{\tilde{p}}(\boldsymbol{\omega}, \boldsymbol{\Omega}, s)=\beta(\boldsymbol{\Omega}) \hat{\tilde{H}}(\boldsymbol{\omega}, s \mid \boldsymbol{\Omega})\left[1+\int \hat{\tilde{\rho}}\left(\boldsymbol{\omega}, \boldsymbol{\Omega}^{\prime}, s\right) d^{2} \boldsymbol{\Omega}^{\prime}\right] .
$$

Integrating Eq. (20) with respect to all possible directions and defining the function,

$$
\hat{\tilde{\rho}}(\boldsymbol{\omega}, s) \equiv \int \hat{\tilde{\rho}}(\boldsymbol{\omega}, \boldsymbol{\Omega}, s) d^{2} \boldsymbol{\Omega},
$$

we get

$$
\hat{\tilde{\rho}}(\boldsymbol{\omega}, s)=[1+\hat{\tilde{\rho}}(\boldsymbol{\omega}, s)] \int \beta(\boldsymbol{\Omega}) \hat{\tilde{h}}(\boldsymbol{\omega}, s \mid \boldsymbol{\Omega}) d^{2} \boldsymbol{\Omega},
$$

which, after defining

$$
\hat{\tilde{h}}(\boldsymbol{\omega}, s) \equiv \int \beta(\boldsymbol{\Omega}) \hat{\tilde{h}}(\boldsymbol{\omega}, s \mid \boldsymbol{\Omega}) d^{2} \boldsymbol{\Omega}
$$

yields

$$
\hat{\tilde{\rho}}(\boldsymbol{\omega}, s)=\frac{\hat{\tilde{h}}(\boldsymbol{\omega}, s)}{1-\hat{\tilde{h}}(\boldsymbol{\omega}, s)} .
$$

Notice that Eq. (21) can be rewritten in terms of the function $\hat{\tilde{\rho}}(\boldsymbol{\omega}, s)$ defined in Eq. (22) as

$$
\hat{\tilde{p}}(\boldsymbol{\omega}, \boldsymbol{\Omega}, s)=\beta(\boldsymbol{\Omega}) \hat{\tilde{H}}(\boldsymbol{\omega} \omega, s \mid \boldsymbol{\Omega})[1+\hat{\tilde{\rho}}(\boldsymbol{\omega}, s)] .
$$

The integration of this equation with respect to all directions yields

$$
\hat{\tilde{p}}(\boldsymbol{\omega}, s)=\hat{\tilde{H}}(\boldsymbol{\omega}, s)[1+\hat{\tilde{\rho}}(\boldsymbol{\omega}, s)],
$$

where [cf. Eq. (12)]

$$
\hat{\tilde{p}}(\boldsymbol{\omega}, s)=\int \hat{\tilde{p}}(\boldsymbol{\omega}, \boldsymbol{\Omega}, s) d^{2} \boldsymbol{\Omega},
$$

and

$$
\hat{\tilde{H}}(\boldsymbol{\omega}, s) \equiv \int \beta(\boldsymbol{\Omega}) \hat{\tilde{H}}(\boldsymbol{\omega}, s \mid \boldsymbol{\Omega}) d^{2} \boldsymbol{\Omega}
$$

Substituting Eq. (24) into Eq. (25) we finally obtain

$$
\hat{\tilde{p}}(\omega, s)=\frac{\hat{\tilde{H}}(\omega, s)}{1-\hat{\tilde{h}}(\omega, s)},
$$

Equation (28) constitutes a generalization of the MontrollWeiss equation [40-42] for the three-dimensional continuous time random walk with independent directions.

\section{AN ISOTROPIC AND UNIFORM WALK}

We next assume that the walker moves inside an isotropic medium so that the pausing time density $\psi(t, \boldsymbol{\Omega})=\psi(t)$ and the probability $\Psi(t \mid \boldsymbol{\Omega})=\Psi(t)$ are both independent of the direction $\boldsymbol{\Omega}$. We additionally assume that inside any sojourn the motion is uniform with a constant speed given by $c$. Thus, after each sojourn the velocity of the walker takes a different direction but with the same modulus $c$ (i.e., after each collision the kinetic energy is conserved). Despite its simplicity the model describes the motion of noninteracting particles-as, for instance, photons-suffering elastic scattering with fixed centers randomly distributed.

For this model the densities $h(\mathbf{r}, t \mid \boldsymbol{\Omega})$ and $H(\mathbf{r}, t \mid \boldsymbol{\Omega})$, respectively, describing the displacement inside a given sojourn in direction $\boldsymbol{\Omega}$ are given by

$$
h(\mathbf{r}, t \mid \boldsymbol{\Omega})=\delta(\mathbf{r}-c t \mathbf{u}) \psi(t),
$$

and

$$
H(\mathbf{r}, t \mid \boldsymbol{\Omega})=\delta(\mathbf{r}-c t \mathbf{u}) \Psi(t),
$$

where $\mathbf{u}$ is the unit vector pointing in direction $\boldsymbol{\Omega}=(\theta, \varphi)$; that is

$$
\mathbf{u}=(\sin \theta \cos \varphi, \sin \theta \sin \varphi, \cos \theta) .
$$

The Fourier transforms of these densities read

$$
\tilde{h}(\boldsymbol{\omega}, t \mid \boldsymbol{\Omega})=\psi(t) e^{i(\boldsymbol{\omega} \cdot \mathbf{u}) c t},
$$

and

$$
\tilde{H}(\boldsymbol{\omega}, t \mid \boldsymbol{\Omega})=\Psi(t) e^{i(\boldsymbol{\omega} \cdot \mathbf{u}) c t} .
$$

We next assume, as we did in the previous section, that after each scattering the new direction is randomized independently of any previous direction [cf. Eq. (19)]. We take in addition a further step and suppose complete isotropy so that

$$
\beta\left(\boldsymbol{\Omega} \mid \boldsymbol{\Omega}^{\prime}\right)=\beta(\boldsymbol{\Omega})=\frac{1}{4 \pi} .
$$

In other words, all outgoing directions are equally likely.

The average,

$$
\tilde{h}(\boldsymbol{\omega}, t)=\int \tilde{h}(\boldsymbol{\omega}, t \mid \boldsymbol{\Omega}) \beta(\boldsymbol{\Omega}) d^{2} \boldsymbol{\Omega}
$$

gives the joint characteristic function of displacement and duration inside any sojourn independent of the direction. In the isotropic case and for uniform motion [cf. Eqs. (31), (33), and (11)] we have

$$
\begin{aligned}
\tilde{h}(\boldsymbol{\omega}, t) & =\frac{1}{4 \pi} \psi(t) \int e^{i(\boldsymbol{\omega} \cdot \mathbf{u}) c t} d^{2} \boldsymbol{\Omega} \\
& =\frac{1}{2} \psi(t) \int_{0}^{\pi} e^{i|\boldsymbol{\omega}| c t \cos \theta} \sin \theta d \theta .
\end{aligned}
$$


After integrating we get

$$
\tilde{h}(\omega, t)=\psi(t) \frac{\sin |\omega| c t}{|\omega| c t},
$$

and analogously,

$$
\tilde{H}(\omega, t)=\Psi(t) \frac{\sin |\omega| c t}{|\omega| c t},
$$

We can now obtain the PDF for the position of the random walker to be at $\mathbf{r}$ at time $t$. We will get such a distribution by means the Fourier-Laplace transform $\hat{\tilde{p}}(\boldsymbol{\omega}, s)$ as given in Eq. (28) in terms of the Fourier-Laplace transform of the densities $h$ and $H$. To this end we need to specify the form of the waiting time density $\psi(t)$. We thus assume that the random instants of time at which the scattering process occurs are distributed according to the Poisson distribution, which constitutes one of the simplest and most universal assumptions. This implies that time intervals inside any sojourn are exponentially distributed [55]

$$
\psi(t)=\lambda e^{-\lambda t} \text { and } \Psi(t)=e^{-\lambda t},
$$

and we have

$$
\tilde{h}(\boldsymbol{\omega}, t)=\lambda e^{-\lambda t} \frac{\sin |\boldsymbol{\omega}| c t}{|\boldsymbol{\omega}| c t}, \quad \tilde{H}(\boldsymbol{\omega}, t)=\frac{1}{\lambda} \tilde{h}(\boldsymbol{\omega}, t) .
$$

We next take the Laplace transform of these expressions. Recalling that [56]

$$
\mathcal{L}\left\{\frac{\sin |\omega| c t}{t}\right\}=\arctan \left(\frac{|\omega| c}{s}\right),
$$

and the property $\mathcal{L}\left\{e^{-\lambda t} f(t)\right\}=\hat{f}(\lambda+s)$, we get

$$
\hat{\tilde{h}}(\omega, s)=\frac{\lambda}{|\omega| c} \arctan \left(\frac{|\omega| c}{\lambda+s}\right),
$$

and

$$
\hat{\tilde{H}}(\omega, s)=\frac{1}{|\omega| c} \arctan \left(\frac{|\omega| c}{\lambda+s}\right) .
$$

Substituting Eqs. (36) and (37) into Eq. (28) we finally obtain

$$
\hat{\tilde{p}}(\omega, s)=\frac{\arctan [|\omega| c /(\lambda+s)]}{|\omega| c-\lambda \arctan [|\omega| c /(\lambda+s)]},
$$

which constitutes the solution of the homogeneous and isotropic model and our starting point for deriving the threedimensional telegrapher's equation. It is worth mentioning that a similar expression was obtained some years ago by Claes and Van den Broeck [57] in the context of modeling the end-to-end distance of polymer chains, although they used a different approach.

\section{TELEGRAPHER'S EQUATION IN THREE DIMENSIONS}

Let us recall that the homogeneous and isotropic random walk described above is a microscopic model of transport in which the particle moves on a straight line until it elastically collides with centers of force randomly distributed in a given region of the three-dimensional space. We will now zoom out this microscopic description and perform the so-called fluid limit, that is, we will rewrite the model for long times and distances $[48,58]$. Because of Tauberian theorems $[59,60]$, long times and distances $t \rightarrow \infty$ and $|\mathbf{r}| \rightarrow \infty$ correspond to small Laplace and Fourier variables $s \rightarrow 0$ and $|\omega| \rightarrow 0$.

We next take the fluid limit in the expression (38) for the transformed PDF of the isotropic walk. We first perform the long-distance limit $(|\omega| \rightarrow 0)$ and briefly postpone the long-time limit $(s \rightarrow 0)$. As $|\omega| \rightarrow 0$ we have the following expansion:

$$
\begin{aligned}
\arctan \left(\frac{|\omega| c}{\lambda+s}\right) & =\frac{|\omega| c}{\lambda+s}-\frac{1}{3}\left(\frac{|\omega| c}{\lambda+s}\right)^{3}+O\left(|\omega|^{5}\right) \\
& =\frac{|\omega| c}{(\lambda+s)^{3}}\left[(\lambda+s)^{2}-\frac{1}{3}(|\omega| c)^{2}+O\left(|\omega|^{4}\right)\right] .
\end{aligned}
$$

Substituting into Eq. (38) and simple algebra yield

$$
\hat{\tilde{p}}(\omega, s)=\frac{(\lambda+s)^{2}-(|\omega| c)^{2} / 3+O\left(|\omega|^{4}\right)}{s(\lambda+s)^{2}+\lambda(|\omega| c)^{2} / 3+O\left(|\omega|^{4}\right)} .
$$

In the long-time limit $(s \rightarrow 0)$ this expression may be written as [61]

$$
\hat{\tilde{p}}(\omega, s)=\frac{\lambda^{2}+2 \lambda s+O\left(s^{2},|\omega|^{2}\right)}{\lambda^{2} s+2 \lambda s^{2}+\lambda(|\omega| c)^{2} / 3+O\left(s^{3},|\omega|^{4}\right)} .
$$

We, therefore, propose as a fluid limit approximation the following expression for the PDF of the uniform and isotropic walk:

$$
\hat{\tilde{p}}(\omega, s)=\frac{\lambda / 2+s}{\lambda s / 2+s^{2}+|\omega|^{2} c^{2} / 6} .
$$

Let us next derive the three-dimensional TE. To this end we begin with Eq. (41) and try to find an associated differential equation satisfied by $p(\mathbf{r}, t)$. In this way we multiply both sides of Eq. (41) by the denominator and rewrite the result as

$$
s^{2} \hat{\tilde{p}}(\omega, s)-s+\frac{\lambda}{2}[s \hat{\tilde{p}}(\omega, s)-1]=-\frac{c^{2}}{6}|\omega|^{2} \hat{\tilde{p}}(\omega, s) .
$$

We now proceed with Fourier inversion. Taking into account

$$
\mathcal{F}^{-1}\left\{|\boldsymbol{\omega}|^{2} \hat{\tilde{p}}(\boldsymbol{\omega}, s)\right\}=-\nabla^{2} \hat{p}(\mathbf{r}, s), \quad \mathcal{F}^{-1}\{1\}=\delta(\mathbf{r}),
$$

we see that

$$
s^{2} \hat{p}(\mathbf{r}, s)-s \delta(\mathbf{r})+\frac{\lambda}{2}[s \hat{p}(\mathbf{r}, s)-\delta(\mathbf{r})]=\frac{c^{2}}{6} \nabla^{2} \hat{p}(\mathbf{r}, s) .
$$

We next address Laplace inversion. With the standard initial conditions,

$$
p(\mathbf{r}, 0)=\delta(\mathbf{r}),\left.\quad \frac{\partial p(\mathbf{r}, s)}{\partial t}\right|_{t=0}=0,
$$

and the Laplace inversion formulas [56],

$$
\begin{aligned}
\mathcal{L}^{-1}\left\{s^{2} \hat{p}(\mathbf{r}, s)-s \delta(\mathbf{r})\right\} & =\frac{\partial^{2} p(\mathbf{r}, t)}{\partial t^{2}}, \\
\mathcal{L}^{-1}\{s \hat{p}(\mathbf{r}, s)-\delta(\mathbf{r})\} & =\frac{\partial p(\mathbf{r}, t)}{\partial t},
\end{aligned}
$$

we see that $p(\mathbf{r}, t)$ satisfies the three-dimensional TE,

$$
\frac{\partial^{2} p}{\partial t^{2}}+\frac{1}{\tau} \frac{\partial p}{\partial t}=v^{2} \nabla^{2} p
$$


with $\tau=2 / \lambda$ as the characteristic time and $v=c / \sqrt{6}$ as the characteristic speed.

We remark that the TE enjoys both wave and diffusion characteristics. This duality becomes even more apparent as time progresses. Thus, as $t \rightarrow 0$ Eq. (43) reduces to the wave equation whereas as $t \rightarrow \infty$ it does to the diffusion equation. Indeed, scaling time with $\tau$ one can easily see that [51]

$$
\frac{\partial^{2} p}{\partial t^{2}} \simeq v^{2} \nabla^{2} p \quad(t \rightarrow 0), \quad \frac{\partial p}{\partial t} \simeq D \nabla^{2} p \quad(t \rightarrow \infty),
$$

$\left(D=v^{2} \tau\right)$. Note that this leads to

$$
\left\langle|\mathbf{r}(t)|^{2}\right\rangle \sim t^{2} \quad(t \rightarrow 0), \quad\left\langle|\mathbf{r}(t)|^{2}\right\rangle \sim t \quad(t \rightarrow \infty),
$$

showing the transition from ballistic motion to diffusive motion as time increases.

\section{THE FRACTIONAL ISOTROPIC WALK}

We recall that the second major objective of this paper consists of deriving a three-dimensional fractional telegrapher's equation out of a microscopic picture based on three-dimensional random walks. We proceed as we did in the one-dimensional case [50] and first obtain a fractional generalization of the homogeneous and isotropic walk of the previous section.

To this end we first describe an alternative way of deriving the expression for $\hat{\tilde{p}}(\boldsymbol{\omega}, s)$ in the fluid limit approximation that we have obtained previously [cf. Eq. (41)]. The starting point of such an alternative derivation consists of realizing that the approximation given by Eq. (41) is achieved readily after substituting into Eq. (28) the following expansions in the fluid limit:

$$
\hat{\tilde{h}}(\omega, s)=1-\frac{s}{\lambda}-2\left(\frac{s}{\lambda}\right)^{2}-\frac{1}{3 \lambda^{2}}|\omega|^{2} c^{2} \cdots,
$$

and

$$
\hat{\tilde{H}}(\omega, s)=\frac{1}{\lambda}\left(1+\frac{2 s}{\lambda}\right) \ldots
$$

$(s,|\omega| \rightarrow 0)$. The derivation of these approximate expansions goes as follows. From Eqs. (36) and (39) we write

$$
\hat{\tilde{h}}(\omega, s)=\frac{\lambda}{(\lambda+s)^{3}}\left[(\lambda+s)^{2}-\frac{1}{3}|\omega|^{2} c^{2}+O\left(|\omega|^{4}\right)\right] .
$$

Simple manipulations yield

$$
\begin{aligned}
1-\hat{\tilde{h}}(\omega, s) & =\frac{1}{(\lambda+s)^{3}}\left[s(\lambda+s)^{2}+\frac{\lambda}{3}|\omega|^{2} c^{2}+O\left(|\omega|^{4}\right)\right] \\
& =\frac{1}{(\lambda+s)^{3}}\left[\lambda^{2} s+2 \lambda s^{2}+\frac{\lambda}{3}|\omega|^{2} c^{2}+O\left(s^{3},|\omega|^{4}\right)\right],
\end{aligned}
$$

which when $s \rightarrow 0$ we approximate as

$$
1-\hat{\tilde{h}}(\omega, s) \simeq \frac{1}{\lambda^{3}}\left[\lambda^{2} s+2 \lambda s^{2}+\frac{\lambda}{3}|\omega|^{2} c^{2}\right],
$$

so that

$$
1-\hat{\tilde{h}}(\omega, s) \simeq \frac{s}{\lambda}+2\left(\frac{s}{\lambda}\right)^{2}+\frac{1}{3 \lambda^{2}}|\omega|^{2} c^{2},
$$

which agrees with Eq. (44). Starting from Eq. (37) and following the same approximation scheme we get

$$
\begin{aligned}
\hat{\tilde{H}}(\omega, s) & =\frac{1}{(\lambda+s)^{3}}\left[(\lambda+s)^{2}-\frac{1}{3}|\omega|^{2} c^{2}+O\left(|\omega|^{4}\right)\right] \\
& =\frac{1}{(\lambda+s)^{3}}\left[\lambda^{2}+2 \lambda s-\frac{1}{3}|\omega|^{2} c^{2}+O\left(s^{2},|\omega|^{4}\right)\right] \\
& \simeq \frac{1}{\lambda^{3}}\left(\lambda^{2}+2 \lambda s\right)=\frac{1}{\lambda}\left(1+\frac{2 s}{\lambda}\right) .
\end{aligned}
$$

which is Eq. (45). Substituting Eqs. (45) and (46) into Eq. (28) immediately leads to the fluid limit expression for $\hat{\tilde{p}}(\boldsymbol{\omega}, s)$ as given by Eq. (41).

In order to propose a fractional generalization of the isotropic walk we reproduce the steps of the derivation of the fractional persistent random walk that we did in one dimension [50]. Thus looking at Eq. (44) we propose the following expansion for the sojourn density in the fluid limit:

$$
\hat{\tilde{h}}(\omega, s)=1-(T s)^{\alpha}-2(T s)^{2 \alpha}-\frac{1}{3}(L|\omega|)^{2 \gamma} \ldots
$$

$(s,|\omega| \rightarrow 0)$, where $0<\alpha \leqslant 1,0<\gamma \leqslant 1$ and $T>0$ and $L>0$ are arbitrary parameters. $T$ defines a characteristic time, and $L$ defines a characteristic length.

In addition to the fractional approximation for $\hat{\tilde{h}}(\boldsymbol{\omega}, s)$ we also need to assume a fractional expansion for the function $\hat{\tilde{H}}(\omega, s)$ consistent with Eq. (47). To this end, we return to Sec. II and multiply both sides of Eq. (8) by $\beta(\boldsymbol{\Omega})$ and integrate over all directions $\boldsymbol{\Omega}$, we have

$$
\int_{\mathbb{R}^{3}} h(\mathbf{r}, t) d^{3} \mathbf{r}=\psi(t)
$$

where

$$
h(\mathbf{r}, t)=\int h(\mathbf{r}, t \mid \boldsymbol{\Omega}) \beta(\boldsymbol{\Omega}) d^{2} \boldsymbol{\Omega},
$$

and

$$
\psi(t)=\int \psi(t \mid \boldsymbol{\Omega}) \beta(\boldsymbol{\Omega}) d^{2} \boldsymbol{\Omega}
$$

are the sojourn PDFs independent of direction. Note that in terms of the Fourier transform $\tilde{h}(\omega, t)$ Eq. (48) can be written as $\tilde{h}(\omega=0, t)=\psi(t)$.

Starting from Eq. (9) and following an identical reasoning we may write $\tilde{H}(\omega=0, t)=\Psi(t)$. In the Fourier-Laplace space we thus have

$$
\hat{\tilde{h}}(\boldsymbol{\omega}=0, s)=\hat{\psi}(s) \text { and } \hat{\tilde{H}}(\boldsymbol{\omega}=0, s)=\hat{\Psi}(s) .
$$

However, from Eq. (7) we see that $\hat{\Psi}(s)=[1-\hat{\psi}(s)] / s$, consequently,

$$
\hat{\tilde{H}}(\omega=0, s)=\frac{1}{s}[1-\hat{\tilde{h}}(\omega=0, s)] .
$$

Introducing Eq. (47) into this expression yields

$$
\hat{\tilde{H}}(\omega=0, s)=T^{\alpha} s^{\alpha-1}+2 T^{2 \alpha} s^{2 \alpha-1},
$$

which leads us to conjecture the following fluid limit approximation:

$$
\hat{\tilde{H}}(\omega, s) \simeq T^{\alpha} s^{\alpha-1}+2 T^{2 \alpha} s^{2 \alpha-1} \ldots
$$


$(s \rightarrow 0,|\omega| \rightarrow 0)$. Let us stress that this is simply a conjecture because the approximation given by Eq. (49) might have depended on $\boldsymbol{\omega}$ as well [50].

Substituting Eqs. (47) and (49) into Eq. (28) and some reorganization of terms yield

$$
\hat{\tilde{p}}(\omega, s)=\frac{2 T^{2 \alpha} s^{\alpha-1}\left[s^{\alpha}+1 / 2 T^{\alpha}\right]}{2 T^{2 \alpha}\left[s^{2 \alpha}+s^{\alpha} / 2 T^{\alpha}+|\omega|^{2 \gamma}\left(L^{2 \gamma} / 6 T^{2 \alpha}\right)\right]} .
$$

That is,

$$
\hat{\tilde{p}}(\omega, s)=\frac{s^{\alpha-1}\left(s^{\alpha}+1 / \tau\right)}{s^{2 \alpha}+s^{\alpha} / \tau+v^{2}|\omega|^{2 \gamma}},
$$

where

$$
\tau \equiv 2 T^{\alpha}, \quad v \equiv \frac{1}{\sqrt{6}}\left(L^{\gamma} / T^{\alpha}\right)
$$

$(0<\alpha \leqslant 1,0<\gamma \leqslant 1)$. The parameters $\tau$ and $v$ can be considered as a fractional time and a fractional speed, respectively.

Equation (50) determines the probability distribution of the fractional three-dimensional isotropic random walk in the fluid limit approximations and is the starting point for deriving the fractional telegrapher's equation in three dimensions.

\section{FRACTIONAL TELEGRAPHER'S EQUATION IN THREE DIMENSIONS}

We next proceed to derive the three-dimensional FTE from the fractional isotropic walk. To this end we first need some formalism concerning fractional derivatives.

The Caputo fractional derivative of order $\beta>0$ of a function $\phi(t)$ is defined by the functional [46,47,58,62,63],

$$
\frac{\partial^{\beta} \phi(t)}{\partial t^{\beta}}= \begin{cases}\frac{1}{\Gamma(n-\beta)} \int_{0}^{t} \frac{\phi^{(n)}\left(t^{\prime}\right) d t^{\prime}}{\left(t-t^{\prime}\right)^{1+\beta-n}}, & n-1<\beta<n, \\ \phi^{(n)}(t), & \beta=n\end{cases}
$$

$(n=1-3, \ldots)$. Using this definition the Laplace transform of the Caputo derivative is found to be $[51,63]$

$$
\mathcal{L}\left\{\frac{\partial^{\beta} \phi(t)}{\partial t^{\beta}}\right\}=s^{\beta} \hat{\phi}(s)-s^{\beta-1} \phi(0)-\sum_{j=1}^{n-1} s^{\beta-1-j} \phi^{(j)}(0)
$$

( $n=1-3, \ldots ; n-1<\beta<n)$, where $\mathcal{L}\{\cdot\}$ stands for the Laplace transform and $\hat{\phi}(s)=\mathcal{L}\{\phi(t)\}$.

The second type of fractional derivative that we need is the Riesz-Feller Laplacian of order $\beta(0<\beta \leqslant 2)$ of a function $g(\mathbf{r})$ such that $g(\mathbf{r}) \rightarrow 0$ as $|\mathbf{r}| \rightarrow \infty$. There are several equivalent ways to define it [63], although one of the simplest and most operative definitions is obtained using Fourier analysis. We thus define [58]

$$
\nabla^{\beta} g(\mathbf{r})=\mathcal{F}^{-1}\left\{-|\omega|^{\beta} \tilde{g}(\boldsymbol{\omega})\right\}
$$

$(0<\beta \leqslant 2)$, where $\mathcal{F}^{-1}\{\cdot\}$ stands for the inverse Fourier transform and

$$
\tilde{g}(\boldsymbol{\omega})=\int_{\mathbb{R}}^{3} e^{i \omega \cdot \mathbf{r}} g(\mathbf{r}) d^{3} \mathbf{r}
$$

is the direct transform.
We can now proceed with our derivation of the threedimensional FTE. The first step is to rewrite Eq. (50) as

$$
\left(s^{2 \alpha}+\frac{1}{\tau} s^{\alpha}+v|\omega|^{2 \gamma}\right) \hat{\tilde{p}}(\boldsymbol{\omega}, s)=s^{2 \alpha-1}+\frac{1}{\tau} s^{\alpha-1} .
$$

Having in mind the definition of the Riesz-Feller derivative given by Eq. (54) and recalling that $\mathcal{F}^{-1}\{1\}=\delta(\mathbf{r})$, the Fourier inversion yields

$$
\left(s^{2 \alpha}+\frac{1}{\tau} s^{\alpha}-v^{2} \nabla^{2 \gamma}\right) \hat{p}(\mathbf{r}, s)=\left(s^{2 \alpha-1}+\frac{1}{\tau} s^{\alpha-1}\right) \delta(\mathbf{r}),
$$

which we rewrite as

$$
s^{2 \alpha} \hat{p}(\mathbf{r}, s)-s^{2 \alpha-1} \delta(\mathbf{r})+\frac{1}{\tau}\left[s^{\alpha} \hat{p}(\mathbf{r}, s)-s^{\alpha-1} \delta(\mathbf{r})\right]=v^{2} \nabla^{2 \gamma} \hat{p} .
$$

Taking into account Eq. (53) and the initial conditions on $p(\mathbf{r}, t)$ given by Eq. (42), we have proved in Ref. [50] the following transformation formulas $(0<\alpha \leqslant 1)$ :

$$
\frac{\partial^{2 \alpha} p}{\partial t^{2 \alpha}}=\mathcal{L}^{-1}\left\{s^{2 \alpha} \hat{p}(\mathbf{r}, s)-s^{2 \alpha-1} \delta(\mathbf{r})\right\},
$$

and

$$
\frac{\partial^{\alpha} p}{\partial t^{\alpha}}=\mathcal{L}^{-1}\left\{s^{\alpha} \hat{p}(\mathbf{r}, s)-s^{\alpha-1} \delta(\mathbf{r})\right\} .
$$

Returning to Eq. (55) and taking the inverse Laplace transform we immediately get

$$
\frac{\partial^{2 \alpha} p}{\partial t^{2 \alpha}}+\frac{1}{\tau} \frac{\partial^{\alpha} p}{\partial t^{\alpha}}=v^{2} \nabla^{2 \gamma} p,
$$

which is the fractional telegrapher's equation in three dimensions and constitutes the second major objective of this paper. Let us recall that in Eq. (56) $\tau$ is a fractional time and $v$ is a fractional velocity [cf. Eq. (51)].

We have seen in Sec. IV that the ordinary telegrapher's equation (43) enjoys both wave and diffusion characteristics. We now extend this duality to the FTE. To this end we first take the limit $\tau \rightarrow 0$ in Eq. (56) and let $v \rightarrow \infty$ such that $\tau v^{2} \rightarrow$ $D$ finite. This results in the fractional diffusion equation [cf. Eq. (6)],

$$
\frac{\partial^{\alpha} p}{\partial t^{\alpha}}=D \nabla^{2 \gamma} p
$$

In the same way as the ordinary $\mathrm{TE}$, the fractional equation leads to the fractional diffusion equation (57) as $t \rightarrow \infty$ [50]. Furthermore, the FTE (56) also contains the so-called "fractional wave equation" as a limiting case. Indeed, letting $\tau \rightarrow \infty$ [i.e., $T \rightarrow \infty$, cf. Eq. (51)] and at the same time $L \rightarrow \infty$ such that the fractional velocity $v=L^{\gamma} / T^{\alpha} \sqrt{6}$ remains finite, Eq. (56) reduces to a wavelike equation,

$$
\frac{\partial^{2 \alpha} p}{\partial t^{2 \alpha}}=v^{2} \nabla^{2 \gamma} p .
$$

Since when $\alpha=1 / 2$ and $\gamma=1$ this equation reduces to an ordinary diffusion equation, we follow Mainardi [46] and call Eq. (58) the "fractional diffusion-wave equation." We finally mention that the fractional diffusion-wave equation (58) is the short-time limit of the FTE (56) regardless the value of the time constant $\tau[50]$. 
All of this reflects the fact that the FTE embraces two different dynamics: One, at short times, representing fractional wavelike behavior, and another, at long times, representing a fractional diffusionlike behavior. This constitutes the fractional generalization of the dual character between waves and diffusions showed by the ordinary TE.

\section{CONCLUDING REMARKS}

In this paper we have obtained the three-dimensional telegrapher's equation (ordinary and fractional) out of a random walk model. The model is a three-dimensional generalization of the persistent random walk on the line. It is based on multistate random walks with a continuum in the number of states representing the different spatial directions that the walker can take. In the special case of homogeneous and isotropic walks the general equations can be solved exactly in the Fourier-Laplace space. This model seems to be rather appropriate for the transport of particles undergoing elastic scattering with fixed centers of force randomly distributed. We have shown that in the fluid limit (i.e., long times and distances) the master equation for the PDF of the homogeneous and isotropic random walk is the three-dimensional telegrapher's equation with characteristic time and velocity related to the corresponding microscopic parameters of the random walk model.

We also have generalized the telegrapher's equation to account for anomalous transport in three dimensions. To this end we have extended the homogeneous and isotropic random walk to allow for fractional behavior. We have thus seen that the master equation for the fractional walk is the fractional telegrapher's equation in three dimensions. As in the one-dimensional case [50], an exact solution for the characteristic function $p(\omega, t)$ of the three-dimensional FTE (56) can be obtained along with some asymptotic and approximate expressions. However, for the sake of simplicity and briefness, we have not treated them here, and we will deal with these questions in a future work. In any case, we address the interested reader to the works of Mainardi and collaborators $[46,47,62,64]$ on solutions for fractional diffusion and fractional wave-diffusion equations and to Orsingher and collaborators [65-67] on several kinds of solutions to the FTE.

Like in one dimension, we also have shown that the FTE accounts for two different dynamics. One of them at short times when the FTE reduces to the fractional wave-diffusion equation and a second one when at long times the FTE reduces to the fractional diffusion equation. This dual character is even more manifest for the time-fractional equation where in Eq. (56) the spatial exponent $\gamma=1$ and only time is fractional. In this case, one can see easily that the the mean square displacement exists and its evaluation goes along the same lines as in the one-dimensional case [50] which results in the following exact expression:

$$
\left\langle|\mathbf{r}(t)|^{2}\right\rangle=2 v^{2} t^{2 \alpha} E_{\alpha, 2 \alpha+1}\left(-t^{\alpha} / \tau\right)
$$

where $E_{\alpha, 2 \alpha+1}(z)$ is the two-parameter Mittag-Leffler function (see Refs. [50,66] for further details). From this exact expression it easily is shown that the mean square displacement exists and that is approximated by

$$
\left\langle|\mathbf{r}(t)|^{2}\right\rangle \sim t^{2 \alpha} \quad(t \rightarrow 0) ; \quad\left\langle|\mathbf{r}(t)|^{2}\right\rangle \sim t^{\alpha} \quad(t \rightarrow \infty),
$$

which clearly shows that in the course of time the fractional telegraph process suffers transitions between different anomalous diffusion regimes depending on the value of the fractional exponent $\alpha$.

Let us finally remark that we have exclusively dealt with three-dimensional problems and that the formalism presented cannot be used for any dimension, at least, in a direct way. Indeed, the mathematical treatment of Sec. III (and, therefore, all subsequent developments) must be adapted to the dimension considered. In transport problems one does not expect dimensions greater than three, and the question, besides some theoretical interest, is rather irrelevant from the practical point of view. This is not, however, the case for two dimensions where transport on surfaces has a great number of applications in, for example, semiconductor electronics [68], chemical physics [69], or biophysics [70], just to name a few. The extension of the formalism to include two-dimensional walks is under current investigation.

\section{ACKNOWLEDGMENTS}

Partial financial support from MINECO under Contract No. FIS2016-78904-C3-2-P and from AGAUR under Contract No. 2014SGR608 is acknowledged.
[1] G. H. Weiss, Physica A 311, 381 (2002).

[2] J. J. Duderstadt and W. R. Martin, Transport Theory (Wiley, New York, 1979).

[3] M. Shlesinger, J. Klafter, and G. Zumofen, Fractals 03, 491 (1995).

[4] D. J. Durian and J. Rudnick, J. Opt. Soc. Am. A 14, 235 (1997).

[5] P. A. Lemieux, M. U. Vera, and D. J. Durian, Phys. Rev. E 57, 4498 (1998).

[6] D. J. Durian and J. Rudnick, J. Opt. Soc. Am. A 16, 837 (1999).

[7] D. D. Joseph and L. Preziosi, Rev. Mod. Phys. 61, 41 (1989); 62, 375 (1990).

[8] A. J. Ishimaru, Appl. Opt. 28, 2210 (1989).

[9] J. Masoliver and G. H. Weiss, Eur. J. Phys. 17, 190 (1996).
[10] J. M. Porrà, J. Masoliver, and G. H. Weiss, Phys. Rev. E 55, 7771 (1997).

[11] J. B. Keller, Proc. Natl. Acad. Sci. USA 101, 1120 (2004).

[12] M. Abdel-Aziz and S. Gavin, Phys. Rev. C 70, 034905 (2004).

[13] J. Dunkel, P. Talkner, and P. Hänggi, Phys. Rev. D 75, 043001 (2007).

[14] D. Jou, J. Casas-Vázquez, and G. Lebon, Extended Irreversible Thermodynamics, 4th ed. (Springer, Berlin, 2010).

[15] V. A. Cimmelli, A. Sellitto, and D. Jou, Phys. Rev. B 81, 054301 (2010).

[16] V. Méndez, D. Campos, and W. Horsthemke, Phys. Rev. E 90, 042114 (2014)

[17] J. Fort and V. Méndez, Phys. Rev. Lett. 82, 867 (1999). 
[18] J. Masoliver, K. Lindenberg, and G. H. Weiss, Physica A 157, 891 (1989).

[19] S. I. Heizler, Nucl. Sci. Eng. 166, 17 (2010).

[20] A. V. Plyukhin, Phys. Rev. E 81, 021113 (2010).

[21] S. Goldstein, Q. J. Mech. Appl. Math 4, 129 (1951).

[22] J. Masoliver, J. M. Porrà, and G. H. Weiss, Physica A 182, 593 (1992).

[23] J. M. Porrà, J. Masoliver, and G. H. Weiss, Physica A 218, 229 (1995).

[24] S. Godoy and L. S. García-Colín, Phys. Rev. E 55, 2127 (1997).

[25] M. Boguñá, J. M. Porrà, and J. Masoliver, Phys. Rev. E 58, 6992 (1998).

[26] A. D. Kolesnik and E. Orsingher, J. Appl. Prob. 42, 1168 (2005).

[27] E. Orsingher and A. De Gregorio, J. Theor. Prob. 20, 769 (2007).

[28] A. Kolesnik and M. A. Pinsky, J. Stat. Phys. 142, 828 (2011).

[29] J. Masoliver, Phys. Rev. A 45, 2256 (1992).

[30] S. Havlin and D. Ben-Avraham, Adv. Phys. 36, 695 (1987).

[31] J. P. Bouchaud and A. Georges, Phys. Rep. 195, 127 (1990).

[32] R. Metzler and J. Klafter, Phys. Rep. 339, 1 (2000).

[33] B. J. West, M. Bologna, and P. Grigolini, Physics of Fractal Operators (Springer, Berlin/New York, 2003).

[34] R. Metzler and J. Klafter, J. Phys. A 37, R161 (2004).

[35] R. Balescu, Aspects of Anomalous Transport in Plasmas (Taylor \& Francis, London, 2005).

[36] J. Klafter and I. Sokolov, Phys. World 18, 29 (2005).

[37] I. I. Eliazar and M. F. Shlesinger, Phys. Rep. 527, 101 (2013).

[38] B. J. West, Rev. Mod. Phys. 86, 1169 (2014).

[39] B. J. West, Fractional Calculus View of Complexity: Tomorrow's Science (CRC, Boca Raton, FL, 2016).

[40] E. W. Montroll and G. H. Weiss, J. Math. Phys. 6, 167 (1965).

[41] E. W. Montroll and M. F. Shlesinger, in Studies in Statistical Mechanics, edited by J. L. Lebowitz and E. W. Montroll (NorthHolland, Amsterdam, 1984), Vol. 11.

[42] G. H. Weiss, Aspects and Applications of the Random Walk (North-Holland, Amsterdam, 1994).

[43] H. Scher and E. W. Montroll, J. Stat. Phys. 9, 101 (1973).

[44] H. Scher and E. W. Montroll, Phys. Rev. B 12, 2455 (1975).

[45] D. ben-Avraham and S. Havlin, Diffusion and Reactions in Fractals and Disordered Systems (Cambridge University Press, Cambridge, UK, 2000).

[46] F. Mainardi, Appl. Math. Lett. 9, 23 (1996).
[47] F. Mainardi, Y. Luchko, and G. Pagnini, Fract. Calc. Appl. Analysis 4, 153 (2001).

[48] R. Gorenflo, F. Mainardi, and A. Vivoli, Chaos 34, 87 (2007).

[49] A. Rebenshtok, S. Denisov, P. Hänggi, and E. Barkai, Phys. Rev. E 90, 062135 (2014).

[50] J. Masoliver, Phys. Rev. E 93, 052107 (2016).

[51] J. Masoliver and K. Lindenberg, Eur. Phys. J. B 90, 107 (2017).

[52] R. G. Gordon, J. Chem. Phys. 44, 1830 (1966).

[53] I. Burshtein, A. A. Zharikov, and S. I. Temkin, J. Phys. B 21, 1907 (1988).

[54] A. G. Kofman, R. Zaibel, A. M. Levine, and Y. Prior, Phys. Rev. A 41, 6434 (1990).

[55] J. F. C. Kingman, Poisson Processes (Oxford University Press, Oxford, 2002).

[56] G. E. Roberts and H. Kaufman, Table of Laplace Transforms (Saunders, Philadelphia, 1966).

[57] I. Claes and C. Van den Broeck, J. Stat. Phys. 49, 383 (1987).

[58] R. Balescu, Chaos 34, 62 (2007).

[59] W. Feller, An Introduction to Probability Theory and its Applications (Wiley, New York, 1971), Vol. II.

[60] R. A. Handelsman and J. S. Lew, SIAM J. Math. Anal. 5, 425 (1974).

[61] Note that in the denominator we keep terms of higher order in $s$ and $\omega$ than in the numerator. This is necessary for the stability of the Fourier-Laplace inversion (see Ref. [59]).

[62] R. Gorenflo and F. Mainardi in Fractals and Fractional Calculus in Continuum Mechanics, edited by A. Carpinteri and F. Mainardi (Springer, Berlin, 1997).

[63] I. Podlubny, Fractional Differential Equations (Academic, San Diego, 1999).

[64] F. Mainardi and G. Pagnini, J. Comp. Appl. Math. 207, 245 (2007).

[65] E. Orsingher and X. Zhao, Chinese Ann. Math. B 24, 45 (2003).

[66] E. Orsingher and L. Beghin, Probab. Theory Relat. Fields 128, 141 (2004).

[67] M. D’Ovidio, E. Orsingher, and B. Toaldo, Stoch. Anal. Appl. 32, 1009 (2014).

[68] S. Hadsegawa and F. Grey, Surf. Sci. 500, 84 (2002).

[69] H. Bulou and J.-P. Bucher, Phys. Rev. Lett. 96, 076102 (2006).

[70] P. Schuck and H. Zhao, Methods Mol. Biol. 627, 15 (2010). 